\title{
Senkt ein hoher Selbstbehalt die Gesundheitskosten?
}

\author{
Immanuel Lampe
}

\section{Relevanz}

Die Gesundheitskosten in der Schweiz und damit auch die Prämien für Krankenversicherungen steigen jährlich an. In der politischen Debatte werden hohe Franchisen bisweilen als Allheilmittel betrachtet, um die Versicherten zu mehr Kostenbewusstsein anzustossen. Dabei wird immer wieder auf Evidenz zurückgegriffen, welche zeigt, dass eine höhere Franchise tatsächlich die Gesundheitskosten senkt. Aber was wäre gewonnen, wenn die Einsparungen auf Kosten der Qualität der Gesundheitsversorgung geht? Eine neue Forschungsarbeit zeigt, dass die Kosten insbesondere deshalb sinken, weil Versicherte auf durchaus sinnvolle Leistungen verzichten, während sich die Preise für die in Anspruch genommenen Leistungen kaum ändern.

\section{Quelle}

Brot-Goldberg, Zarek C., Amitabh Chandra, Benjamin R. Handel und Jonathan T. Kolstad (2017), What does a Deductible Do? The Impact of Cost-Sharing on Health Care Prices, Quantities, and Spending Dynamics, Quarterly Journal of Economics 132(3), 1261-1318.

Die Ausgaben für die medizinische Versorgung steigen weltweit rasant an. Beispielsweise betrugen 1995 die durchschnittlichen Gesundheitsausgaben pro Einwohner der USA \$320 im Monat. Bis 2015, innerhalb von lediglich 20 Jahren, ist

\footnotetext{
I. Lampe $(\bowtie)$

Universität St.Gallen, St.Gallen, Schweiz

E-Mail: Immanuel.Lampe@unisg.ch
} 
dieser Wert auf mehr als das Doppelte (\$780) angewachsen. Diese Entwicklung ist in anderen hoch entwickelten Ländern, beispielsweise Österreich, der Schweiz oder Deutschland, ähnlich. Wachsen die Ausgaben in den nächsten Jahren weiterhin mit derselben Geschwindigkeit, ist das Gesundheitssystem nur schwer finanzierbar.

Die Gesundheitsausgaben sind in den letzten Jahren explodiert. Im Jahr 2014 betrugen die Kosten für medizinische Versorgung der USA 17 Prozent des BIP.

Die politischen Entscheidungsträger befinden sich in einer Zwickmühle. Auf der einen Seite müssen die Gesundheitsausgaben reduziert werden. Auf der anderen Seite sollte dies nicht die Qualität der medizinischen Versorgung mindern. Das Ziel kann natürlich nicht in der Reduzierung aller medizinischen Behandlungen liegen. Stattdessen sollen der Bevölkerung die Gesundheitskosten stärker bewusst gemacht werden. Ein möglicher Lösungsansatz in der Krankenversicherung ist die verstärkte Verwendung von Franchisen, einer Selbstbeteiligung. Auf diese Weise müssen Versicherte bis zu einer jährlichen Höchstgrenze selbst für alle anfallenden Gesundheitskosten aufkommen. Daten aus den USA zeigen, dass dieses Instrument immer häufiger eingesetzt wird. 2009 hatte nur ca. jeder fünfte Versicherte eine jährliche Franchise von mehr als \$1000. 2014 traf dies bereits auf nahezu jeden zweiten Arbeiternehmer zu.

Ist eine hohe Franchise zielführend? Werden die Versicherten kostengünstigere Ärzte aufsuchen oder nur auf nicht notwendige medizinische Behandlungen verzichten, sodass die Qualität der medizinischen Versorgung unverändert bleibt? Diesen Fragen geht ein Forscherteam um Amitabh Chandra von der Universität Harvard nach. Mehrfach wurde schon nachgewiesen, dass nach Einführung einer Franchise die Gesundheitsausgaben sinken. Allerdings konnte noch kein Forscher die genaue Wirkungskette von Franchisen und ihre Folgen für die Nachfrage nach medizinischen Leistungen festmachen.

Um die Auswirkung der Einführung einer hohen Franchise nachzuverfolgen, verwenden die Wissenschaftler Daten eines grossen Arbeitgebers in den USA. Wie in den Vereinigten Staaten üblich waren die Mitarbeiter über ihren Arbeitgeber krankenversichert. Das Unternehmen beschäftigte zum Zeitpunkt der Auswertung mehrere 10.000 Mitarbeiter. Die Arbeitnehmer erzielten ausserordentlich hohe Einkommen. Lediglich $8 \%$ verdienten weniger als $\$ 100.000$ pro Jahr. Zuerst sah die Krankenversicherung keine Franchise vor. Aufgrund steigender Gesundheitskosten entschloss sich das Unternehmen allerdings, eine Franchise in Höhe von $\$ 3000$ bis $\$ 4000$ einzuführen. Die Mitarbeiter wurden drei Jahre vor der tatsächlichen Umsetzung darüber informiert. Das Forschungsteam nutzte diese Anpassung, um zu untersuchen, wie die Versicherten auf die Einführung 
einer vergleichsweise hohen Selbstbeteiligung reagieren. Der Datensatz umfasst Angaben zu Kosten und Diagnose jedes einzelnen Arztbesuches im Zeitraum von vier Jahren vor und zwei Jahren nach Einführung der Franchise.

In den vier Jahren vor Einführung der Franchise stiegen die durchschnittlichen jährlichen Gesundheitsausgaben pro Mitarbeiter von ca. \$4000 auf \$5200. Im Jahr der Änderung sanken diese Ausgaben abrupt auf rund $\$ 4400$. Bei einem Vergleich dieser Werte muss man allerdings Inflation und Alterung der Mitarbeiter während des sechsjährigen Beobachtungszeitraums berücksichtigen. Beides erhöht die Gesundheitskosten. Unter Berücksichtigung dieser Faktoren schätzen die Wissenschaftler einen Anstieg der durchschnittlichen Gesundheitskosten in den vier Jahren vor der Einführung der Franchise um ca. $8 \%$. Die Franchise verringerte jene Kosten im Durchschnitt um $18 \%$. Abb. 1 illustriert diese Entwicklung anhand der monatlichen Gesundheitskosten. Auf der horizontalen Achse bezeichnen $t_{-4}$ bis $t_{-1}$ die Jahre vor sowie $t_{0}$ und $t_{1}$ die Jahre während bzw. nach der Anpassung. Die dunkelblaue untere Linie zeigt den Kostenverlauf unter Berücksichtigung von Inflation und Alterung. Die graue Linie zeigt die Entwicklung der nominalen Kosten.

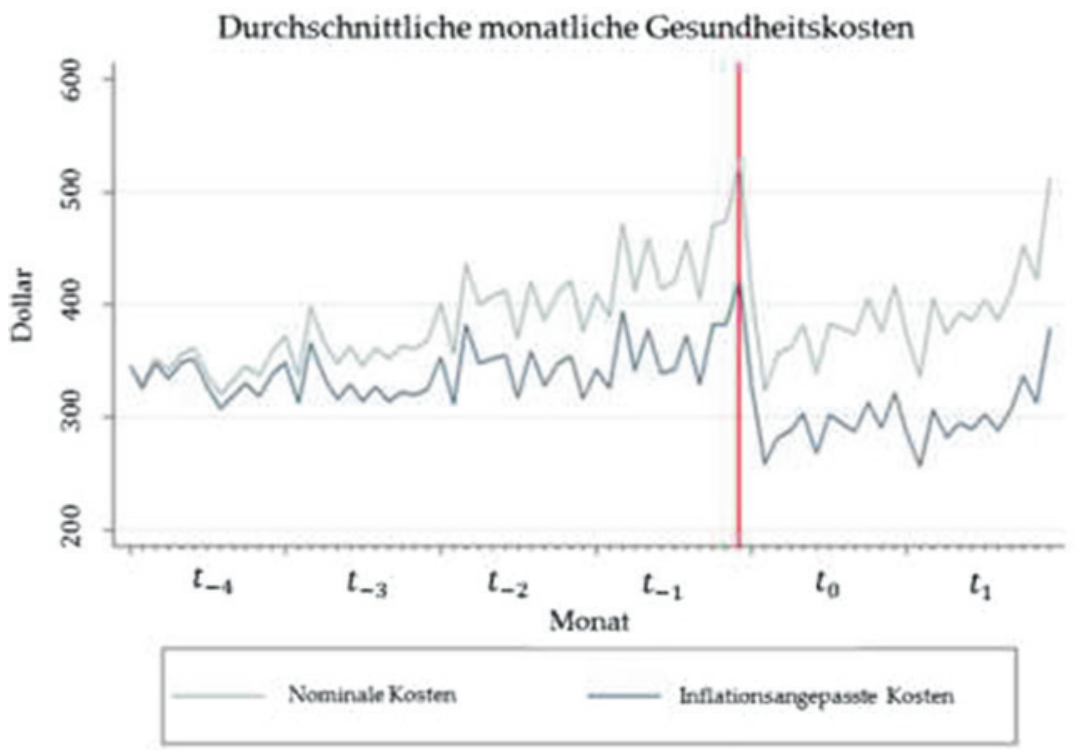

Abb. 1 Monatliche Gesundheitskosten bei Einführung einer Franchise (Quelle: BrotGoldberg u. a., 2017, S. 13) 
Wodurch wird der beschriebene Kostenrückgang verursacht? Die Wissenschaftler nennen drei mögliche Gründe. Erstens setzen sich Versicherte bewusster mit der Wahl eines Arztes auseinander. Ihnen wurde beispielsweise ein OnlinePreisvergleichstool empfohlen, das auf kostengünstigere Ärzte hinweist. Zweitens ersetzen Versicherte teure Behandlungsmethoden durch kostengünstigere. Drittens nehmen Versicherte generell weniger Behandlungen in Anspruch. Das Forscherteam kann zeigen, dass der einzige Grund für den Kostenrückgang in der geringeren Inanspruchnahme medizinischer Leistungen liegt. Letztere geht ebenfalls um $18 \%$ zurück. Eine solche Entwicklung wäre unproblematisch, solange nur unwichtige Leistungen gekürzt wurden. Allerdings verzichten die Versicherten auch auf vorbeugende Behandlungen wie etwa Vorsorgeuntersuchungen.

Die jährlichen Kosten für die medizinische Versorgung der Mitarbeiter sinken nach der Einführung der Franchise um 18 Prozent. Einzige Ursache hierfür ist der teilweise Verzicht auf medizinische Leistungen in der gesamten Bandbreite.

Selbst bei den Kränksten beobachten die Wissenschaftler einen deutlichen Kostenrückgang. Somit nahmen auch jene Menschen, für welche die medizinische Versorgung am wichtigsten ist, weniger Leistungen in Anspruch. Dies trat ein, obwohl die Gesundheitskosten dieser Gruppe den Selbstbehalt ohnehin übersteigen und die Kosten weiterer Behandlungen von der Krankenversicherung getragen werden. Ein Beispiel mag den Zusammenhang verdeutlichen: Ein Diabetiker muss regelmässig zu Kontrolluntersuchungen und benötigt täglich Medikamente. Die monatlichen Kosten dafür betragen \$400. Bis zum Ende des Jahres summieren sich diese Kosten auf $\$ 4800$ und überschreiten die maximale Franchise. Zu Beginn des Jahres erkrankt der Versicherte an einer starken Grippe. Ein Arztbesuch würde zu Kosten von \$200 führen. Nachdem der Selbstbehalt bereits anderweitig überschritten ist, fallen die Kosten nicht beim Patienten, sondern bei der Versicherung an, und sollten daher die Entscheidung zum Arztbesuch nicht mehr beeinflussen. Die Ergebnisse der Studie widersprechen dem allerdings und zeigen, dass auch Kranke mit hohen Gesamtkosten ihre Arztbesuche verringern.

Solange die Versicherten damit rechnen, dass sie über das gesamte Jahr die Franchise nicht überschreiten werden, müssen sie jede weitere Ausgabe vollständig selber zahlen. Die Entscheidung, ob sie eine medizinische Leistung in 
Anspruch nehmen sollen, hängt damit vor allem von jenen Kosten $a b$, die sie selbst zum Zeitpunkt der Behandlung bezahlen müssen. Ob mit einer aktuellen Ausgabe am Ende des Jahres die Franchise ausgeschöpft wird, sodass diese Kosten am Ende von der Versicherung übernommen und nicht selbst getragen werden, beeinflusst die aktuelle Entscheidung nicht. Liegen die innerhalb des Jahres bisher angefallenen Kosten unterhalb der Franchise, muss ein Versicherter in seiner Wahrnehmung die zusätzlichen Behandlungskosten selbst tragen. Die empirischen Ergebnisse zeigen, dass die medizinischen Behandlungen in diesem Fall um nahezu die Hälfte zurückgingen. Dies bedeutet, dass im Vergleich zu den Jahren vor Einführung der Franchise jeder zweite Arztbesuch ersatzlos gestrichen wurde.

Wenn jedoch die bisher angefallenen Kosten bereits vorzeitig den Selbstbehalt übersteigen, erkennen die Versicherten, dass sie ab diesem Zeitpunkt für weitere Behandlungen nicht mehr selbst aufkommen müssen, sondern die Krankenversicherung alle zusätzlichen Kosten übernimmt. Die Studie zeigt, dass sich die Versicherten in diesen Fällen ähnlich verhielten wie vor Einführung der Selbstbeteiligung. Sie verzichteten nicht mehr auf medizinische Behandlungen, nachdem die Höhe der Franchise erreicht wurde.

Wenn die Versicherten damit rechnen, dass sie über das Jahr den Betrag der Franchise nicht überschreiten und damit die Kosten von medizinischen Behandlungen selber tragen müssen, nehmen sie nur noch nahezu die Hälfte der üblichen Leistungen in Anspruch.

Die Ergebnisse legen nahe, dass die Einführung einer hohen Franchise kein nachhaltiger Ansatz ist, um die Gesundheitskosten zu verringern. Einzige Ursache für die Reduzierung der Gesamtkosten ist der Verzicht auf medizinische Leistungen. Dies betrifft die komplette Bandbreite des Angebots. Versicherte kürzen sowohl unwichtige als auch wichtige Behandlungen. Es ist nicht auszuschliessen, dass dieses Verhalten zu verspäteten Diagnosen und somit zu hohen Folgekosten führt. Zudem sollte man berücksichtigen, dass es sich bei den betrachteten Personen um Spitzenverdiener handelt. Der negative Einfluss einer hohen Franchise könnte bei Normal- bzw. Geringverdienern noch stärker ausfallen. 
Open Access Dieses Kapitel wird unter der Creative Commons Namensnennung 4.0 International Lizenz (http://creativecommons.org/licenses/by/4.0/deed.de) veröffentlicht, welche die Nutzung, Vervielfältigung, Bearbeitung, Verbreitung und Wiedergabe in jeglichem Medium und Format erlaubt, sofern Sie den/die ursprünglichen Autor(en) und die Quelle ordnungsgemäß nennen, einen Link zur Creative Commons Lizenz beifügen und angeben, ob Änderungen vorgenommen wurden.

Die in diesem Kapitel enthaltenen Bilder und sonstiges Drittmaterial unterliegen ebenfalls der genannten Creative Commons Lizenz, sofern sich aus der Abbildungslegende nichts anderes ergibt. Sofern das betreffende Material nicht unter der genannten Creative Commons Lizenz steht und die betreffende Handlung nicht nach gesetzlichen Vorschriften erlaubt ist, ist für die oben aufgeführten Weiterverwendungen des Materials die Einwilligung des jeweiligen Rechteinhabers einzuholen.

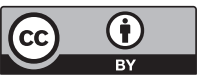

